\title{
Hacía una ciudadanía radical para la reforma de la democracia: ciudadano y ámbitos de ciudad ${ }^{1}$
}

Makes a radical citizenship for the reform of democracy: citizen and city areas

\author{
German Zuluaga Ramírez ${ }^{2}$
}

\section{RESUMEN}

El presente artículo propone una reflexión sobre el concepto de democracia radical en los ámbitos de la de ciudad y ciudadanía. Ello, en los marcos de la perspectiva cultural. El autor aborda tales perspectivas desde autores como Chantal Mouffe y Jurguen Habermas

\section{Palabras clave:}

Ámbitos de ciudad, ciudadano, ciudadanía radical para la reforma de la democracia

\begin{abstract}
This article proposes a reflection on the concept of radical democracy in the areas of city and citizenship. This, within the framework of the cultural perspective. The author addresses such perspectives from authors such as Chantal Mouffe and Jurguen Habermas
\end{abstract}

\section{Key words:}

Areas of city, citizen, radical citizenship for the reform of democracy

\footnotetext{
${ }^{1}$ Artículo presentando en el marco del seminario sobre debates y perspectivas en torno a la democracia, ciudadanía y los derechos, Facultad de ciencias jurídicas de la Universidad del Atlántico. Año 2016.

${ }^{2}$ Filósofo y Abogado en Formación de la Universidad del Atlántico. Asistente de Investigación perteneciente al Grupo de Investigación Cronotopias. Correo: gzuluagar21@gmail.com

ISSN: 2346-2787

Recibido: 1 de Marzo de 2018

Aprobado: 28 de Abril de 2018.
} 


\section{Introducción}

Es claro la etapa crítica que atraviesa la praxis democrática mundial, la cual ha afectado todas las dimensiones existenciales del ser humano, fenómeno que lleva a Takis Fotopoulos (2015) a indicar que la crisis de la democracia tiene un carácter multidimensional, de la que no se escapa ningún ámbito social, siendo la causa fundamental la concentración desmedida del poder.

Por tal motivo, es necesario redireccionar las caminos reflexivos de la teorización política, económica, social y ecológica actual, pues lo problemas que surgen tienen rasgos cada vez más complejos. La meta inicial es negar la concentración del poder anteriormente denunciada, además de impulsar la superación de los obstáculos intelectuales que impiden un mejoramiento de la praxis sociopolítica democrática, las cuales provienen de la tradición socialista, capitalista, histórica, y que tiene en sus reductos contemporáneos formas para mantenerse, como son los movimientos ecológicos, feministas, autonomistas, homosexuales, entre otros.

Lo que se debe buscar es la distribución igualitaria entre los ciudadanos en el aspecto político y económico, a través de una democracia directa en el primero caso, y una democratización de la economía con un alcance más social y ecológico.

El desarrollo de este producto parte de la tesis titulada Por una Democracia Inclusiva: Ciudadano, Educación y Pensamiento Crítico para una Reforma Política (2017), con la que se abordó múltiples perspectivas analíticas compartidas entre crisis democráticas y democracia inclusiva. 
Precisamente, son variados los enfoques que abordan la crisis de la democracia y una posible salida de ella, por lo que es importante, y beneficioso, encontrar puntos en común entre los diferentes proyectos intelectuales; es decir, crear una mixtura metodológica que logre constituir un diagnóstico social, político y económico que ayude a conocer los síntomas que empujan al fracaso el proyecto democrático actual, y sólo a través de los síntomas se puede elaborar una solución.

Ante esta razón, lo que se va a exponer a continuación busca mostrar al concepto de ciudadanía como un elemento fundamental para que la praxis de la democracia sea viable, debido a que el ciudadano es la voz y acción directa que afecta a la democracia.

La participación y el discernimiento activo de la ciudadanía es una condición sine qua non para la legitimización de la democracia. Una comunidad democrática podría concebirse como aquella donde los ciudadanos tienen acceso a los medios y al conocimiento, por los cuales pueden defender sus derechos frente a sus dirigentes, y de la misma manera pueden hacer propuestas para el desarrollo de su entorno colectivo.

Al respecto, Shklar (1997 cit. Zuluaga, 2017, p. 3) define "que ciudadano es aquel, que por su propia voluntad y sin intermediaciones somete su libertad natural para aceptar un conjunto de reglas que lo sitúan en igualdad de condiciones que el resto de la comunidad", pero que también toma decisiones colectivas que afectan a la comunidad y las decisiones de la comunidad afectan al sujeto-ciudadano.

En consecuencia, este escrito indagará sobre la ciudadanía en su acto de toma de decisiones colectivas, y este como elemento básico para conseguir una reforma política; por ende, el escrito consta de tres partes. 
La primera parte se referirá al ciudadano dese una mirada culturao, en tanto protagonista fundante en el funcionamiento satisfactoria de la democracia, ya que de él se desprende el contenido y la expresión del bienestar socio-político de la colectividad.

La segunda parte se tocará el tema de las esferas pública y privada, desde la perspectiva de Jurgen Habermas y Hannah Arendt, para llegar después a la perspectiva de los ámbitos propuestos por Takis Fotopoulos.

La última parte tratará el concepto de ciudadanía desde un enfoque radical de la democracia, que parte de los planteamientos de Chantal Mouffe.

Este escrito, como se dijo anteriormente, viene de la tesis de grado en Filosofía de la Universidad del Atlántico, producto de una labor hermenéutica, utilizada de manera crítica para evitar arbitrariedades y confusiones conceptuales del contenido que se adquiera de los autores escogidos.

\section{El ciudadano desde una perspectiva cultural.}

Un horizonte de la acción política en la actualidad, es proponer espectros gnoseológicos en los que el ser humano que vive en comunidad pueda hablar, oír y callar, y esto para que logre construir diálogos sociales e interculturales en un mapa colectivo que se caracteriza por la facultad que tiene todo sujeto de ser diferente.

El sujeto social debe ser aprehendido como un individuo político que es atravesado por un lenguaje que busca construir una identidad que manifieste lo que es como ser. El lenguaje "no está allí a la disposición, y no surge ante los requerimientos lexicales, sintácticos o gramaticales, pues se trata de un "saber" más 
allá de lo que las meras elucubraciones sobre la lengua hacen aparecer en la superficie" (Albano y Naughton, 2005, p. 18). El sujeto es un ser humano genérico, en el que se amalgama, siguiendo a Edgar Morin (2000), el homo sapiens, homo ludens, homo faber, homo economicus, entre otros, por lo que es emocional y se puede ir más allá de la simple argumentación, o entendimiento racional.

Por consiguiente, el sujeto es un "consumidor con un comportamiento emocional y aclamador y la comunicación pública se disuelve en actitudes, siempre estereotipadas, de recepción aislada" (Matellart, 1997, p. 57) en la que siempre buscará un Otro como encuentro de él mismo. Es decir, en su constante búsqueda por develarse.

Conforme a esto, el sujeto busca encontrase y desarrollarse en el Otro, en la que pueda reconocer y reconocerse en un entramado cultural con el que pueda situarse en el espectro de lo público, o sea, como ciudadano, ya que sólo dentro del mapa de la colectividad es que puede existir un ciudadano, pues lo que convierte al sujeto en ciudadano es la oportunidad de tomar decisiones colectivas profundas.

El ciudadano solo será ciudadano, dirá Hannah Arendt (1993) en La Condición Humana:

cuando este puede ser visto y oído por otros, donde se deriva su significado del hecho de que todos ven y oyen desde una posición diferente. Este es el significado de la vida pública, comparada con la cual incluso la más rica y satisfactoria vida familiar solo puede promover la prolongación o multiplicación de la posición de uno con sus acompañantes aspectos y perspectivas. Solo donde las cosas pueden verse por muchos en una variedad de aspectos y sin cambiar su identidad, de manera que quienes se agrupan a su alrededor sepan que ven lo mismo en total diversidad, solo allí aparece auténtica y verdaderamente la realidad mundana (p. 66).

Entonces, este ciudadano que se observa en una ciudadanía, busca la igualdad en la desigualdad de la ciudadanía, pues el rasgo público de la ciudadanía, es la 
diversidad y provocar las particularidades de los sujetos que la conforman, ya que lo que se desea es legitimar la idea de libertad, autonomía y heteronomía, en la que las rivalidades desaparezcan y que los vacíos y quiebres de la democracia se reparen con las diferencias ciudadanas.

El concepto de ciudadano puede remitir al de identidad, es posible que el "concepto de ciudadano debe darse: en el nivel político (expresado en la práctica política), en el de las condiciones económicas; el status de ciudadano se adquiere a través de complejos procesos de socialización que inculcan en las personas el sentido de pertenencia con la sociedad" (Valderrama y Sorbes, 2011, p. 22).

Luis Alberto Sánchez (2009) dice en Esfera Pública y Esfera Privada que:

En las sociedades contemporáneas el ciudadano subyace a las demás identidades y permite manejar los conflictos que pueden surgir entre quienes profesan diferentes ideologías porque pueden servir para cultivar la virtud política de conciliación responsable de los intereses en conflicto (p. 19).

Sin embargo, los conceptos de ciudadanía y de ciudadano deben salirse del aspecto formal y legal, para sumergirse en la idea plena de ciudad como espacio de acción cultural. Es decir, que el ciudadano debe remitir aquel que no solo habita la ciudad sino que la consume como elemento nutricio de conocimiento e identificación. Así que lo legitimo sería forjar al ciudadano en cómo llevar la vida cotidiana en el sentido que habitamos lo habitual de nuestro mundo.

Avanzando en el razonamiento, el ciudadano es experiencia vivida en ciudad, lo que invita a que sea visto de una aísthesis ${ }^{3}$, con lo que se rescata el saber intuitivo,

\footnotetext{
${ }^{3}$ La aisthesis, rescata la capacidad perceptiva por medio de un conocimiento intuitivo, sensible, que no es menos significativo que el conocimiento conceptual. Desde esta perspectiva, la obra de arte en el espacio público debe estar concebida de tal manera que provoque una experiencia estética no sólo de los expertos, los entendidos en las reglas del arte y su historia. Tomado de la estética de la recepción en la obra de arte urbana de Alberto Vargas Rodríguez. Conferencia dada en el $1^{\circ}$ congreso de filosofía colombiana realizado en la Universidad Jorge Tadeo Lozano en Bogotá. 2006.
} 
sensible, sin menoscabar ese conocimiento conceptual tan preponderante, pero concebir al ciudadano desde un bloque pleno de experiencias transforma al sujeto en el espacio público y privado es que su existencia es vital, y que su presencia es experiencia social, pues "el ciudadano no es medio hacia un fin sino un fin en sí mismo para el ente mismo de su ciudadanía, que es la ciudad. Siendo concreto, las políticas públicas que permiten la participación del sujeto tienen que percibirse a partir de la ciudad desde el ciudadano. La ciudad debe forjarse desde el ciudadano y no a lo contrario" (Zuluaga, 2017, p. 30).

El ciudadano es más que una figura nomológica, normalizada y formal, sino una construcción compleja de saberes, racionalidades y emociones, en la que se mezclan todo tipo de accidentes espirituales y racionales.

Pensar el ciudadano como un ser global y variante, que danza con cualquier actividad interna y externa hasta que pueda consolidarse en un espacio que no le secuestre su libertad.

El ciudadano se enfrenta a que "las decisiones que se tomen al respecto a las políticas sociales no deben estar al margen del desarrollo económico" (Aghón, 2001).

En esta dirección, como lo piensa Margarita Rozas (2006) en el texto Democratización, responsabilidad social y derechos sociales:

el desafío esta justamente en la necesidad de pensar la política social, ciudadana incorporada en las decisiones de carácter económico, como así también en la resignificación de los fines respecto de considerarlas como la vía de desarrollo de los derechos sociales y la ciudadanía social (p. 25).

Así que hay que mirar al ciudadano desde su capacidad de asociación y disociación, en la que se convierte en un nosotros. El fin es que el ciudadano 
reconozca su capacidad de ubicar consensos y entendimiento, por tal razón es esencial analizar al ciudadano como actor público y privado, pero también social y ecológico, debido a que es estas esferas y ámbitos es donde se toman las decisiones colectivas y en la que emana la ciudadanía.

\section{Lo público y lo privado, y los ámbitos ciudadanos.}

La pregunta por las esferas sociales o ámbitos ciudadanos es preponderante y necesaria porque es el cuestionamiento que indaga por el ¿Dónde estamos? ¿Cuál es el lugar que ocupamos en la red llamada comunidad? Preguntarse por el espacio es inquirir cómo se devela el ser humano en tanto su libertad, autonomía y emancipación reflexiva, pues es en los espacios sociales en los que se toman las decisiones colectivas. En lugar en el que la democracia fluye.

Es conocida la fórmula planteada por Jürgen Habermas: esfera pública y esfera privada. Para el alemán, la esfera pública es el espacio en el que se origina la opinión pública, la cual no remite a un lugar material, sino a la participación y conjunción de múltiples criterios que incumben a una colectividad social, y en la que se intervienen diferentes temas que tienen que ver con la sociedad. Está idea parte de la concepción opositiva binaria entre el Estado y la esfera privada, que es la que tiene que ver con las relaciones afectivas y racionales entre los integrantes de una familia.

Dice Jürgen Habermas (2004) en Crítica e Historia de la Opinión Pública:

La entrada está fundamentalmente abierta a todos los ciudadanos. En cada conversación en la que los individuos privados se reúnen como público se constituye una porción de espacio público. [...] Los ciudadanos se comportan como público, cuando se reúnen y conciertan libremente, sin presiones y con 
la garantía de poder manifestar y publicar libremente su opinión, sobre las oportunidades de actuar según intereses generales. En los casos de un público amplio, esta comunicación requiere medios precisos de transferencia e influencia: periódicos y revistas, radio y televisión son hoy tales medios del espacio público (p. 61).

La visión de Habermas proviene del rescate que hace de los conceptos de Pólis

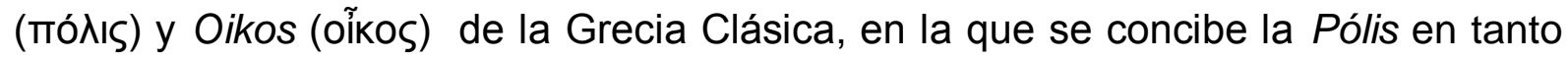
"conjunto de dispositivos simbólicos suficientes para ligar a los sujetos dentro del marco de una sociedad regida por el interés general" (Sanabria, 2011, p. 15), por lo que equivale al uso libre de la expresión en asuntos políticos, y que no pueden ser conducidos por los medios de comunicación, de ahí su crítica a la publicidad ${ }^{4}$.

En el caso de Hannah Arendt (2009), percibe la esfera pública en La Condición Humana como:

Por el contrario, este hecho puede intensificar también el disfrute y consumo de las cosas del mundo, toda clase de intercambios en que el mundo no se considera fundamentalmente como koinon, lo que es común a todos. Sólo la existencia de una esfera pública y la consiguiente transformación del mundo en una comunidad de cosas que agrupa y relaciona a los hombres entre sí, depende por entero de la permanencia. Si el mundo ha de incluir un espacio público, no se puede establecerlo para una generación y planearlo sólo para los vivos, sino que debe superar el tiempo vital de los hombres mortales ( $p$. 63-64).

Es decir, que para Arendt el espacio público es el lugar donde se generan las transformaciones que el mundo necesita, porque es donde los ciudadanos se comunican y deliberan sobre los asuntos que afectan al colectivo. Es decir, dónde se construye la ciudadanía deliberativa.

Ahora bien, la mirada de Habermas como de Arendt queda reducida por la falta de implicaciones culturales, sociales y ecológicas, que son indirectamente 
sumergida en lo público y lo privado, y en la que influye más la emociones que la razón.

Por lo tanto hay que multidimensionar los ámbitos existenciales del ser humano en sociedad, ya que la reforma política exige estar acorde con la crisis multidimensional de la democracia, por lo que la propuesta de Takis Fotopoulos está acorde a las necesidades de una ciudadanía radical y transformadora.

Para Fotopoulos (2005) en Crisis Multidimensional y Democracia Inclusiva:

la democracia entre en el campo social, que supone la creación de instituciones de autogestión en las fábricas, oficinas y lugares generales de producción, así como en instituciones educativas y culturales (medios de comunicación, arte, etc.). Los consejos de trabajadores, consejos de estudiantes y demás, obtienen la autogestión de los lugares de producción y de los centros educativos, guiados por los objetivos generales fijados por las asambleas ciudadanas, así como por las preferencias de los ciudadanos como productores pero también como consumidores (p. 98).

Por lo que la perspectiva de Habermas y Arendt resulta insuficiente para amalgamar las complejidades ciudadanas de los sujetos, ya vistos como actores sociales determinantes. Por ende, el griego propone cuatro ámbitos en los que el ciudadano contemporáneo está tomando decisiones colectivas: el ámbito político (Donde se toma las decisiones políticas), económico (se toman las decisiones económicas a partir de varias opciones), social (decisiones de trabajo, educación y que implique cualquier institución cultural) y ecológico (decisiones que afectan el entorno natural y se establece las relaciones entre naturaleza y sociedad), y que contienen las siguientes ciudadanías:

- La ciudadanía política implica nuevas estructuras políticas y el retorno a la concepción clásica de política (democracia directa). 
- La ciudadanía económica comporta nuevas estructuras económicas de propiedad y control demóticos de los recursos económicos (democracia económica).

- La ciudadanía social supone estructuras de autogestión en el lugar de trabajo, la democracia en el hogar y nuevas estructuras de bienestar social en las que todas las necesidades básicas (determinadas democráticamente) sean satisfechas.

- La ciudadanía cultural implica nuevas estructuras democráticas de divulgación y control de la información y la cultura (medios de comunicación, arte, etc.), que permitan a cada miembro del demos participar en el proceso y al mismo tiempo desarrollar su potencial intelectual y cultural.

De acuerdo con esto, la ciudadanía posee intrínsecamente una pluralidad innegable, convirtiéndose en una unidad fundamental de la acción política, en la que se legitime la libertad y la autonomía.

\section{Por una ciudadanía radical desde la perspectiva de chantal mouffe}

Radical no expresa una rama extremista o beligerante de una pensamiento o una creencia, asimilada como un tipo de intransigencia político social. Lo radical es el principio de una democracia plural. Siguiendo a Chantal Mouffe, "la instauración de un pluralismo agonístico hace falta una nueva interpretación que sea la expresión e la diversidad de las luchas por la igualdad y de la relación que establecen con la libertad" (Mouffe, 1999, p. 23), pero sin excluir a las instituciones como los pilares del funcionamiento de la vida liberal y democrática. 
Por lo que "el ciudadano tiene que ser visto y oído por otros, de lo que deriva su significado, del hecho de que todos ven y oyen desde una posición diferente. Sólo donde las cosas pueden verse por muchos en una variedad de aspectos y $\sin$ cambiar su identidad" (Navarro, 2010, p. 45). De manera que quienes se agrupan a su alrededor sepan que ven lo mismo en total diversidad, sólo allí aparece auténtica y verdaderamente la realidad mundana.

Esta ciudadanía es inclusiva, que no simpatiza con la homogeneidad para sobrevivir, sino que observa en la heterogeneidad una necesidad para legitimar los ideales de igualdad, fraternidad y libertad, los cuales han fracasado, pues el disenso está sustentado en un antagonismo político que emana del proyecto teórico político de Carl Schmitt: amigo/enemigo, el cual pretende aniquilar al enemigo.

El paso a una práctica ciudadana agonística pretende que el otro diferente no sea percibido como un enemigo que debe ser aniquilado o eliminado sino como un adversario, lo que facilitaría establecer límites de respeto y reconocimiento, consolidándo que los ideales de igualdad y libertad sean posible.

En particular, una ciudadanía radical y plural crea "una cadena de equivalencias entre las diversas luchas por la igualdad y el establecimiento de una frontera política en dar nueva identidad" (Mouffe, 1999, p. 24) a la sociedad, y que apunte a una transformación en las relaciones de fuerza y la creación de una nueva y fresca democracia inclusiva.

Por esa razón, es clave repensar la idea de ciudadanía radical como la salvación de la democracia, pues la maximización de la participación del ciudadano en las decisiones estructurales del Estado o Nación, afectará la forma en que percibe el mundo y especialmente el hombre como ser dentro un mundo político. 
Ante este tenor, el poder no debe seguir siendo practicado como jerarquización o relaciones de poder dispersados por toda la estructura social, sino que la meta primordial de una política realmente democrática, no sea, como dice Chantal Mouffe (1999) en Retorno a la Política:

erradicar el poder, sino multiplicar los espacios en los que las relaciones de poder estarán abiertas a la contestación democrática. En la proliferación de esos espacios con vistas a la creación de condiciones de una auténtico pluralismo agonístico, tanto dominio del Estado como en el de la sociedad civil, se inscribe la dinámica inherente a la democracia radical y plural (p. 2425)

Por lo tanto, sólo la colectividad que devenga en igualdad, libertad y autonomía podrá establecer una democracia radical basada en los principios de pluralidad y heteronomía.

Asevera Mouffe (1999):

Entiendo por esto una identificación colectiva con una interpretación democrática radical de los principios del régimen democrático liberal: libertad e igualdad. Semejante interpretación presupone que esos principios se entienden de manera tal que se tome en cuenta las diferentes relaciones sociales y las distintas posiciones subjetivas en que son pertinentes: género, clase, raza, etnicidad, orientación sexual, etc. (p. 103).

En consecuencia, el ideal de ciudadanía es aquel que extienda los principios de igualdad y libertad "combinando el ideal de derechos y pluralismo y las ideas de inspiración pública y preocupación ético-política, una nueva concepción democrática de ciudadanía podría restaurar la dignidad a lo político y proporcionar el vehículo de la construcción de una hegemonía democrática radical” (Mouffe, 1999, p. 105).

Está concepción tendría repercusiones favorables en la aplicación de políticas públicas, porque la divorciaría de la misma política y la conecta con la existencia del hombre como ser que existe. Es decir, que las políticas públicas se sujeten a mecanismos institucionalizados, sino que sean producto de un devenir cotidiano de 
la vida ciudadana, que es donde se definen los parámetros existenciales en que deciden como conducir las vidas en tanto integrantes de una sociedad.

\section{Referencias.}

Arendt, H. (1993). La condición Humana. Barcelona Editorial Paídos.

Foutopulos, T (1997). Democracia Inclusiva. Barcelona. Editorial Gedisa.

Fotopoulos, T. (2005). Crisis Multidimensional y Democracia Inclusiva. Cataluña. Editorial GADil.

Habermas, Jürgen. (2004). Crítica e historia de la opinión pública. Barcelona. Editorial Gustavo Gili.

Mouffe, C. (1999). El retorno de lo político. Barcelona. Editorial Paidos.

Navarro, L. (2010). Entre esferas públicas y ciudadanía: las teorías de Arendt, Habermas y Mouffe. Barranquilla. Editorial Uninorte.

Rozas, M. (2006). Democratización, responsabilidad social y derechos sociales. En: N. Burgos (Coord). Acerca de la democracia y los derechos sociales: política social y trabajo social. Buenos Aires. Espacio Editorial.

Sanabria, F. (2011). De tramoyeros a tramoyistas. Consideraciones para pasar de una moral rural a una ética civil. Ediciones Universidad Nacional de Colombia. Bogotá. 
Sklar, J. (1997). Justicia y ciudadanía. En J. Afichard, \& J. De Foucauld, Pluralismo y equidad: la justicia social en las democracias y los derechos sociales y trabajo social. Buenos Aires. Espacio Editorial. 